\title{
Treatment Options in Vulvar Lichen Sclerosus: A Scoping Review
}

\author{
Nilanchali Singh $^{1}$, Neha Mishra ${ }^{2,3}$, Prafull Ghatage ${ }^{1}$ \\ 1. Gynecologic Oncology, Tom Baker Cancer Centre, Calgary, CAN 2. Obstetrics and Gynecology, All India Institute of \\ Medical Sciences, New Delhi, Greater Noida, IND 3. Obstetrics and Gynecology, Government Institute of Medical \\ Sciences, Greater Noida, IND
}

Corresponding author: Nilanchali Singh, nilanchalisingh@gmail.com

\begin{abstract}
Vulvar lichen sclerosus (VLS) is a chronic inflammatory disorder, which affects women of all ages. The aim of this review is to focus on first-line, second-line, and maintenance therapies as well as follow-up of women with VLS. With numerous controversies, we decided to conduct a scoping review on this subject. A review protocol was developed, and the Knowledge Resource Services website was used to run a search of articles pertaining to VLS with keywords "Vulvar," "Vulval," and "Lichen Sclerosus." The search was limited to published data from the last 10 years, i.e., July 2009 onward, and researches published in English language. A total of 338 articles pertaining to VLS were obtained. Out of this, 62 were original articles related to management of VLS. Effective treatments such as high-potency topical steroids are now the standard of care and first-line treatment. Follow-up may be done every three to six months for the first two years and then at least yearly to ensure adequacy of treatment and encourage compliance. Long-term follow-up in specialist clinics is recommended for women who have persistent complaints, thickened skin, or history of neoplastic lesion. Monitoring young patients yearly is recommended as there are chances of recurrence.
\end{abstract}

Categories: Dermatology, Obstetrics/Gynecology

Keywords: vulvar, vulval, lichen sclerosus, adult, juvenile, treatment, follow-up, review

\section{Introduction And Background}

Vulvar lichen sclerosus (VLS), also known as vulvar dystrophy in the past, is one of the most common pathologies presenting to vulvar clinics. A study reported that incidence rate of lichen sclerosus increased from 7.4 to 14.6 per 100,000 woman-years between 1991 to 2011 [1]. The vague terminologies like leukoplakia, kraurosis, and dystrophy of vulva were prevalent before International Society for the Study of Vulvovaginal Disease (ISSVD) 1975 classification system. Presently, this disease is included in nonneoplastic and non-infectious entities; vulvar dermatoses in ISSVD classification includes this disease entity with vulvar dermatoses, which are non-neoplastic and non-infectious in nature [2]. VLS has bimodal presentation, both in pre-pubertal girls and postmenopausal women. Some cases may present in reproductive group (18-40) females. It has multitude of causative factors such as autoimmune pathologies, hormones, and infections. The labia, perineum, and perianal areas get affected and present as a patchy, thin, glistening, and ivory-white area. The clinical features suffice diagnosis, and biopsy is rarely done [3]. As far as the treatment strategy is concerned, testosterone was the mainstay of treatment in the past, whereas high-potency steroids are now considered the standard therapy. Testosterone was found ineffective in root canal treatment (RCTs) and has unacceptable side effects [4]. Follow-up and maintenance therapies are also not clearly defined. With numerous controversies, we decided to conduct a scoping review on this subject. The focus of this review is on the first-line, second-line, and maintenance therapies as well as on the follow-up of women with VLS. Additionally, the focus will also be on the difference in management of adult and juvenile VLS.

\section{Review}

\section{Methods}

A review protocol was framed. PubMed was explored for the literature search related to VLS with the assistance of Knowledge Resource Services website. Various databases were searched: MEDLINE (Ovid), Evidence-Based Medicine (EBM) Reviews, PubMed, Cumulative Index to Nursing and Allied Health Literature (CINAHL), MEDLINE, and Excerpta Medica Database (EMBASE). Keywords used for search were: "Vulvar," "Vulval," "lichen sclerosus et atrophicus," "kraurosis," "dystrophy," "VIN," “Cancer," and "lichen sclerosus." The published data from July 1999 in English language were accessed. After a comprehensive search, 338 articles concerning to VLS were retrieved. All of these articles were screened; 276 articles were excluded because of the following reasons: not correlated to the objective of this review, i.e., management of VLS, or they were not original articles. This review incorporates 62 studies. Inclusion of original articles and review articles was done on priority basis. In some cases, relevant older data was also included such as role of testosterone in the management of VLS. There are not any papers available regarding testosterone 


\section{Cureus}

usage owing to its severe unpropitious effects. All the articles were retrieved in full text. This review did not seek individual data sources, and a narrative analysis was done. The data was summarized in the form of a descriptive review.

\section{Therapeutic options for vulvar lichen sclerosus}

The various modalities of treatment of VLS are summarized in Table 1.

\begin{tabular}{|c|c|}
\hline S. No. & Treatment Modalities for Vulvar Lichen Sclerosus \\
\hline 1. & Local Steroid Therapy \\
\hline 2. & Intra-lesional Steroid Therapy \\
\hline 3. & Topical Calcineurin Inhibitors \\
\hline 4. & Topical Androgens, Progesterone, and Estrogens \\
\hline 5. & Other Local Pharmacologic Agents Like Retinoids \\
\hline 6. & Emollients \\
\hline 7. & Phototherapy \\
\hline 8. & Laser Therapy \\
\hline 9. & Photodynamic Therapy \\
\hline 10. & Cryotherapy \\
\hline 11. & Fat Grafting \\
\hline 12. & Adipose-Derived Stem Cells and Platelet-Rich Plasma Therapy \\
\hline 13. & Herbal Therapies \\
\hline 14. & Systemic Therapy \\
\hline 15. & Dietary Modification \\
\hline 16. & Surgical Intervention \\
\hline
\end{tabular}

TABLE 1: Treatment Modalities for Vulvar Lichen Sclerosus

1. Steroid Therapy: Local

The gold standard treatment for VLS consists of high-potency topical corticosteroids (TCS), such as clobetasol dipropionate. Clobetasol propionate cream (0.05\%) significantly reduces symptoms and improves skin characteristics $[5,6]$. The most common regimen is to apply it thoroughly over lesion two times daily for three months [7]. As per our experience, we also recommend a constant dosing schedule of twice a day for 12 weeks [5]. Creams are water-based, spread easily, and absorbed easily; however, ointments are oil-based, have less spreadability, and take a longer time to absorb. Ointments are more potent than creams. Creams may contain alcohols or preservatives that are much more likely to cause burning, stinging, and/or irritation, particularly of the inflamed/fissured mucosa in lichen sclerosus, and as such, ointments are recommended over creams as first-line therapy. About $60 \%-70 \%$ of patients experience complete remission of their symptoms [8]. Others, who continue to have flares and remissions after 12 weeks of therapy, are advised to use clobetasol propionate $0.05 \%$ as required [9]. The side effects of TCS include irritation, burning, dryness, hypopigmentation, and dermal atrophy [10]. The adverse effects of stinging, burning, and dryness are most commonly due to the base of the topical steroid rather than the steroid itself; hypopigmentation and dermal atrophy may occur with topical steroid use, particularly to keratinized skin, but these side effects are specifically noted to rarely occur in most long-term studies of topical steroids for treatment of VLS [11].

The less-potent steroids like mometasone furoate $(0.1 \%)$ and triamcinolone are also found to be effective [12]. However, current recommendations favor the initial use of highly potent steroids as first line of therapy with the less-potent steroids used for maintenance.

Some studies have suggested use of different corticosteroids on the basis of severity of disease. They suggest that very severe disease should be treated with clobetasol propionate ointment, moderate to severe disease with betamethasone dipropionate ointment, mild disease with methylprednisolone aceponate ointment, 
and very mild "burnt out" disease with hydrocortisone ointment [5]. Severity was assessed by the degree of hyperkeratosis of the lesion. Objective return of the vulvar skin to normal color and texture should be the aim to titrate dose and duration of treatment. However, Cochrane Reviews states that potency and dose of topical steroid therapy for treatment of VLS needs further evaluation and research [3]. We therefore recommend what most studies suggest, i.e., usage of clobetasone propionate ointment $(0.05 \%)$ for treatment of any degree of VLS. The management strategy of adults with VLS is summarized in Figure 1.

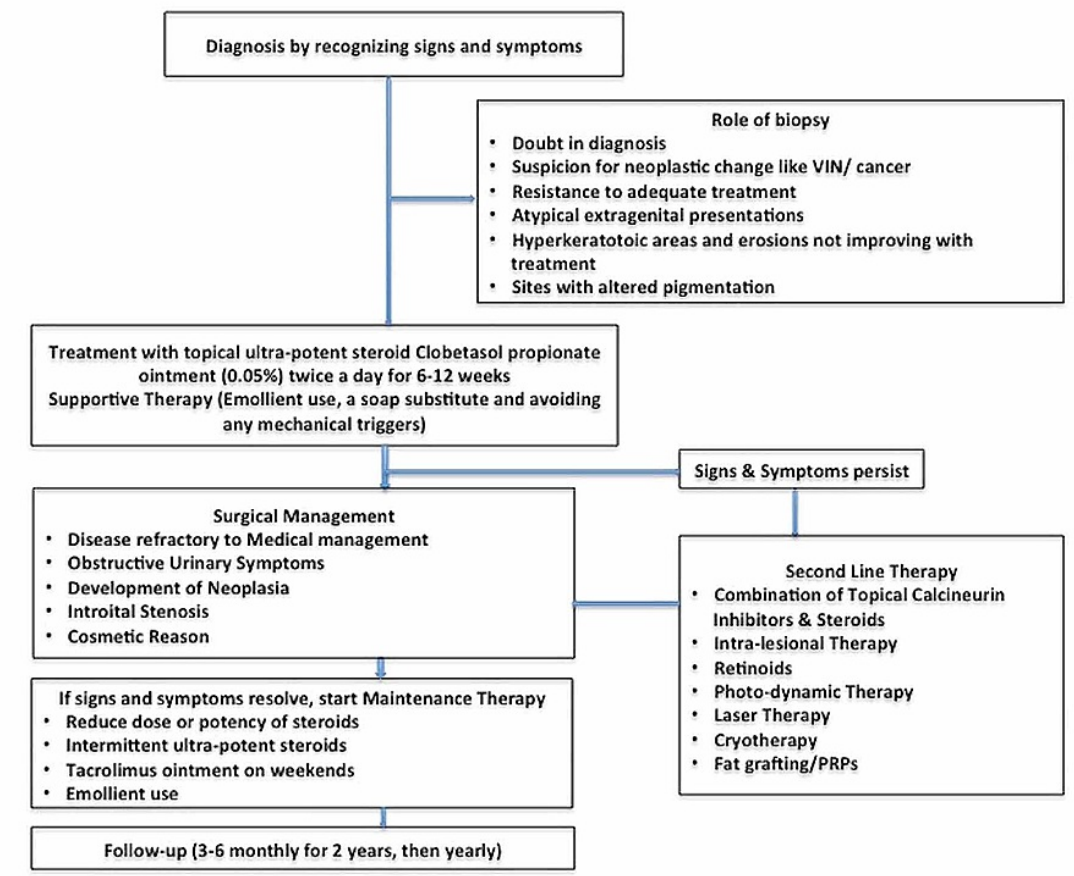

FIGURE 1: Suggested Management of Adult Vulvar Lichen Sclerosus

VIN, Vulvar intraepithelial neoplasia; PRP, platelet-rich plasma.

\section{Intra-lesional Steroid Therapy}

Intra-lesional injection of triamcinolone acetonide has been proposed as an alternative treatment to topical treatment of LS, but again the studies are small [13]. There may be a benefit for intra-lesional steroid injection if it thought that there has been a lack of response to a steroid cream or ointment due to poor penetrance. For thick lichen sclerosus, intra-lesional steroid (triamcinolone $3.3-10 \mathrm{mg} / \mathrm{ml}$ ) may be considered. Pruritic LS has also been treated with TCS or combined corticosteroid and monthly anesthetic/corticosteroid subdermal injection. In a five-year study, time to recurrence was longer with the combined treatment, although women were less satisfied with the injections [14]. Combined treatment can be reserved for women not responding to single therapy. The dose is dependent on the location and thickness of the skin that is being injected, and this can be repeated monthly for two to three months. High steroid doses should not be injected into thin skin or in small areas because the tissue may slough off.

\section{Topical Calcineurin Inhibitors}

The promising effect of calcineurin inhibitors (CIs) on immunomodulation and in blocking the release of inflammatory cytokines has been used to treat VLS widely. Tacrolimis and pimecrolimus are very effective when it comes to treat anogenital LS topically $[15,16]$. However, these findings should be taken with a pinch of salt as the studies included a small number of patients $[15,16]$. They are used as adjunctive therapy for LS but have not been shown to be as effective in initial treatment for LS both clinically and histologically $[17,18]$. These studies have shown the efficacy of using TCIs daily or twice daily in children and adults with partial to complete remission ranging from six weeks to 10 months of treatment $[15,16]$. In one study, complete remission was seen in $43 \%$ and partial remission in $34 \%$ of patients, after using TCIs for 16 weeks [19]. Side effects of TCIs included stinging and burning.

Combining therapy with TCS and TCIs has been studied. An author has reported remission in pediatric cases with LS after alternating clobetasol $0.05 \%$ ointment with tacrolimus ointment [20]. Clobetasol $0.05 \%$ ointment is used as first-line therapy and tacrolimus ointment as a bridging therapy in between the steroid therapy. As patients improved, clobetasol ointment was discontinued, and tacrolimus ointment was used on 
weekends as maintenance therapy. Clearance ranged from four to 156 weeks with an average time of 43.1 weeks. TCI use is recommended in women with corticosteroid-resistant disease or intolerance to steroids. The long-term safety profile of TCIs is not established, and there are concerns about the increased risk of neoplasia, especially with long-term use [7].

\section{Topical Androgens and Progesterone}

Randomized controlled trials have tested and compared testosterone propionate cream (2\%), dihydrotestosterone (DHT) cream (2\%), and progesterone cream with placebo [21,22]. Two authors applied testosterone for three months and a year, respectively, with no significant differences as compared with placebo [21,22]. A relatively small-sized, randomized placebo-controlled trial DHT for three months found no notable difference. So, topical androgens are not justifiable to use in the treatment of VLS [3]. Similarly, topical progesterone was of no benefit [3].

\section{Other Local Pharmacologic Agents}

Retinoids have anti-inflammatory properties and reduce hyperkeratinization. Virgili et al. studied topical tretinoin cream (0.025\%) in a small group of women with histologically proven LS [23]. With four to 13 months of treatment, symptoms, macroscopy, and histology, all improved. Mild-associated skin reactions are found. Pregnancy is a contraindication for their use owing to teratogenic effects reported by them. Borghi et al. reported that tretinoin $0.025 \%$ cream application for six months may represent a useful alternative option to corticosteroids in the treatment of active VLS, acting particularly on hyperkeratosis and pallor [24].

Origoni et al. reported that topical oxatomide has a better relief on itching than placebo due to its antiinflammatory properties. However, it did not have significant effects on other clinical end-points [25].

Shelley et al. hypothesized infectious etiology in vulvar LS and studied antibiotics. They tested penicillin or cephalosporins in an observational study of 15 women not responding to TCS [26]. Symptoms like pain, pruritus, and burning were relieved. Antibiotics, however, should be used only when there are signs of a secondary infection.

Local application of human fibroblast lysate cream, containing anti-inflammatory cytokines as well as wound-healing growth factors, has been found to be useful in treatment [27].

\section{Role of Emollients}

Emollients or moisturizers should be an integral part of treatment of VLS. Emollients have been used to soften and protect the skin from cracks in VLS patients. It is also advisable to use emollient cream as a substitute to soap. Application of moisturizers or paraffin prevents skin contact with urine, which may reduce symptoms. Emollients and lubricants can be used during sexual intercourse, which might otherwise be painful. Long-term maintenance therapy with a moisturizing cream can maintain the symptom relief induced by TCS in women with vulvar LS while being safe and inexpensive. This treatment may also be associated with a reduction in topical corticosteroid use with over half of women being able to eliminate the use of corticosteroids altogether [28]. It should be noted that improvement in symptoms allowing for discontinuation of topical steroids may not correspond to reduction in risk of scarring or development of squamous cell carcinomas (SCC) [5].

\section{Laser Therapy}

Laser therapy has been used in a few small studies for the treatment of VLS refractory to TCS [29,30]. The carbon dioxide laser was used on 40 patients with severe hyperkeratotic VLS not responding to high-potency steroids. Remission was achieved, and their disease was subsequently managed with TCS [29]. The use of fractionally ablative Er:YAG (erbium-doped yttrium aluminum garnet) laser therapy (2940 nm) to a depth of $750 \mu \mathrm{m}$ was evaluated for recalcitrant VLS. At the last follow-up, more than one year after Er:YAG laser treatment, the patient remained asymptomatic on corticosteroid therapy [30].

There is an ongoing trial on the MonaLisa Touch Laser (DEKA Laser, Florence, Italy) looking at the efficacy and safety of the micro-ablative fractional $\mathrm{CO}_{2}$ laser treatment (FxCO2) for VLS (Trial no. NCT03665584). Hopefully, this may throw more light on the role of laser therapy.

\section{Phototherapy}

Ultraviolet A1 (UV-A1) phototherapy has been used for treatment of VLS. UV-A1 phototherapy with initial irradiation intensity of $24 \mathrm{~mW} / \mathrm{cm}^{2}$ and a distance of approximately $25 \mathrm{~cm}$ showed good results; however, later it is revealed that results were inferior to topical steroid treatment [31]. The UV-A1 doses were intensified during the first five treatment sessions and were administered four times per week for a total of 
12 weeks, resulting in 48 irradiations in total. Additional therapy was restricted to the use of emollients. There was significant clinical improvement but was inferior to the current gold standard treatment with topical high-potent corticosteroids. The practicability, relief of itch, and improvement in quality of life are better with corticosteroids. Hence, these options should be reserved for refractory cases and not as first-line therapy.

\section{Photodynamic Therapy}

Photodynamic therapy (PDT) with the use of topical 10\% 5-ALA cream (5-aminolaevulinic acid) has been found to be useful in treating VLS [32]. In a study, after application of freshly prepared 10\% 5-ALA cream with a one-cm margin and incubating for three hours, the lesions were irradiated with $100 \mathrm{~J} / \mathrm{cm}^{2} 633 \mathrm{~nm}$ red light at $100 \mathrm{~mW} / \mathrm{cm}^{2}$ [33]. The same PDT procedure was repeated three times at two-week intervals. ALAPDT is a well-tolerated and effective option for the treatment of VLS. Pain was the main adverse reaction in ALA-PDT. ALA-PDT shows longer remission duration and a higher complete response rate than clobetasol propionate [34]. For patients who relapse after ALA-PDT, steroids can be used as a palliative treatment for those whose symptoms are less severe. If symptoms worsen, ALA-PDT can be administered repeatedly to control recurrent and remaining lesions. Since steroids can alleviate some post-PDT transitory symptoms, a combination of ALA-PDT and corticosteroids might be considered in order to reduce treatment cost [34].

One study reported significant improvement with the use of topical 5-ALA with argon laser light [35]. There is an ongoing trial on the Rivelin ${ }^{\circledR}$ plain patch, which works by forming a protective barrier over a lesion, hence protecting it from further irritation. The premise of the Rivelin ${ }^{\circledR}$ plain patch is a longer pain-free period compared to current treatment with bioadhesive agents, thereby potentially increasing the pain-free period.

\section{Cryotherapy}

Cryotherapy has been used as a treatment modality for VLS refractory to medical management [36]. Temperature of $-186^{\circ} \mathrm{C}$ under general anesthesia has been used in the past to treat VLS. Contact method with one freeze-thaw cycle per lesion and session combined with intra-lesional steroid injection (triamcinolone acetonide) has been used successfully [13]. Postoperative wound care can be done with emollients, compresses, and antiseptic solution. It has been used successfully in children with good acceptance [36]. Cryotherapy may be used as a second-line therapy option in the treatment of long-standing VLS resistant to treatment. However, limiting factors of cryotherapy are a relatively high number of relapses, a long postoperative healing time, and a long time for pain-free status after cryotherapy.

\section{Fat Grafting}

Fat grafting may be considered a supportive or second-line therapy. It may be used in selected cases of VLS not responding to first-line therapy or for anatomical correction to improve sexual function and quality of life [37]. The rationale of this regenerative approach is the presence of stem cell progenitors within adipose tissue capable of differentiating into different mesenchymal tissues and producing anti-inflammatory and immunomodulatory effects. The results of fat grafting are promising; however, further studies are required [38].

\section{Role of Adipose-Derived Stem Cells and Platelet-Rich Plasma Therapy}

Adipose-derived stem cells, now considered as stem cell transplant, are purported to be able to restore and regenerate damaged tissue as in VLS. They have tissue regenerative potential in fibrotic conditions. Adipose-derived stem cells proliferate and differentiate into various mesenchymal tissues. They also have anti-inflammatory and immunomodulatory properties. These factors are hypothesized to ultimately inhibit fibrosis, promote healing, and remodel the extracellular matrix. Similarly, platelet-rich plasma (PRP) therapy acts as a regenerative agent as it has tissue factors for cell growth, migration, repair, proliferation, and regeneration. It is still in experimental stages with few reports of successful management of patients with VLS [39]. Prospective randomized trials are needed to provide an evidence base for this therapy.

\section{Herbal Therapies}

Medicinal plants with their metabolites have been used as a complementary medicine in various inflammatory diseases. Herbal therapies with agents like chamomile, coconut oil, shea butter, aloe vera, calendula, arnica, avocado, etc. [40] have been used in VLS with variable results. The fact that whether they act as emollient or have any therapeutic potential is controversial. Further studies are required to prove their efficacy in treatment of VLS.

\section{Systemic Therapy}

If pruritus persists after corticosteroid and other local therapies, hydroxyzine or doxepin may be given to 
stop nighttime pruritus. In cases of worse pruritus, subcutaneous triamcinolone in the vulvar tissue has been attempted [41]. Acitretin is a retinoid that may be used for lichen sclerosus unresponsive to topical steroids [42]. It is most beneficial for thickened skin due to vulvar sclerosus, which is unresponsive to topical agents. It should not be prescribed if the patient is pregnant or planning for the same, as it is teratogenic.

\section{Role of Dietary Modification}

There are some reports of effect of diet on disease symptomatology and progression of VLS [43]. Pork consumption has been associated with worsening of symptoms in VLS [44]. Other foods advised to be avoided in VLS are spinach, canned and boxed food items, potatoes, nuts, cocoa, beets, turnip, etc. [44]. These items are high in oxalate, which leads to worsening of symptoms in VLS. However, there is no clear evidence for association of these dietary habits with VLS. Some clinicians advocate calcium-rich diet.

\section{Surgical Intervention}

Surgical management of labial agglutination in VLS should be reserved for patients with disease refractory to medical management or with obstructive urinary symptoms. Lysis of adhesion may be required for labial fusions and clitoral hood scarring for cosmetic reasons too. Surgical separation of labial agglutination in postmenopausal women can result in recurrence of labial adhesions and scarring of the tissue. Refractory or recurrent labial agglutination in postmenopausal women can result in symptomatic architectural changes of the vulvovaginal anatomy. In severe introital stenosis, labial lysis of adhesion may not be adequate, and more extensive procedures such as Fenton's operation of a longitudinal incision through the posterior fourchette and perineal body may be required. Surgical separation of tissue can often result in adhesions and increased scarring of the tissue due to recurrence of lichen sclerosus at sites of tissue injury, known as the Koebner phenomenon. Surgical treatment of clitoral phimosis can improve sexual function [45]. Vulval LS recalcitrant to medical treatment or that recurs following simple excision or adhesiolysis may successfully be treated with wider excision and reconstruction with split skin graft (SSG). Oxidized regenerated cellulose has also been studied to prevent recurrence of adhesions [46]. More aggressive surgical treatment like vulvectomy is reserved for women with malignancy or post-inflammatory sequelae [47]. More studies are required as far as surgical management of VLS is concerned.

\section{Maintenance Therapy}

Once the VLS lesion has responded to treatment, maintenance therapy is often required as a preventive measure. Normalcy of skin color and texture is the target, and the dose of topical steroids may be titrated depending upon the outcome. Dosing therapy may vary from thrice a week to daily depending upon the severity. Some advises an intermittent highly active topical steroid agent as and when required [5]. Others believe that patients with moderate to severe lesion may require a less-potent steroid therapy or topical CIs for a long time till complete remission [48]. Individualized preventive TCS regimens used by compliant patients modify the course of the disease. There is a significant difference in symptom control, scarring, and occurrence of vulvar carcinoma between compliant and partially compliant patients [5]. If side effects like atrophy or corticosteroid dermatitis develop, the potency of the TCS should be reduced. If, however, hyperkeratosis returns, the potency of the TCS should be increased. Therefore, there is no fixed dosage regimen that can be followed for maintenance therapy. Long-term maintenance therapy with a moisturizing cream can maintain symptom relief induced by TCS while being safe and inexpensive. Emollient use may also be associated with a reduction in topical corticosteroid use. If one is not responding to treatment, other conditions should be investigated and ruled out, and biopsy should be considered.

\section{Treatment of Juvenile Lichen Sclerosus}

Topical steroids are the mainstay of treatment, just like their adult counterpart. Potent or very potent topical steroids, applied once or twice a day for six to 12 weeks, with progressive weaning, are the treatment of choice. In a case series of pediatric LS, successful treatment was obtained with TCS (betamethasone dipropionate $0.05 \%$ ointment, diflorasone diacetate $0.05 \%$ ointment, or clobetasol propionate $0.05 \%$ ointment) twice daily for six to 8 weeks. Minimal side effects were observed [49]. While mid-potency TCS, such as triamcinolone acetonide or mometasone furoate, have been found to be effective in pediatric LS, most current recommendations do not support their use for first-line treatment. Supportive therapy is in the form of emollient use and a soap substitute. If symptoms resolve, TCS potency can be gradually reduced, and maintenance therapy can be started. A combination therapy including hydrocortisone $1 \%$ ointment and methylprednisolone aceponate $0.1 \%$ ointment is recommended for maintenance. Low-concentration topical tacrolimus has been shown to be an effective and safe treatment for children with anogenital lichen sclerosus [50]. VLS usually improves at puberty but can persist into adulthood. Monitoring the patients yearly is recommended as there are chances of recurrence. The management strategy of juvenile VLS is summarized in Figure 2. 


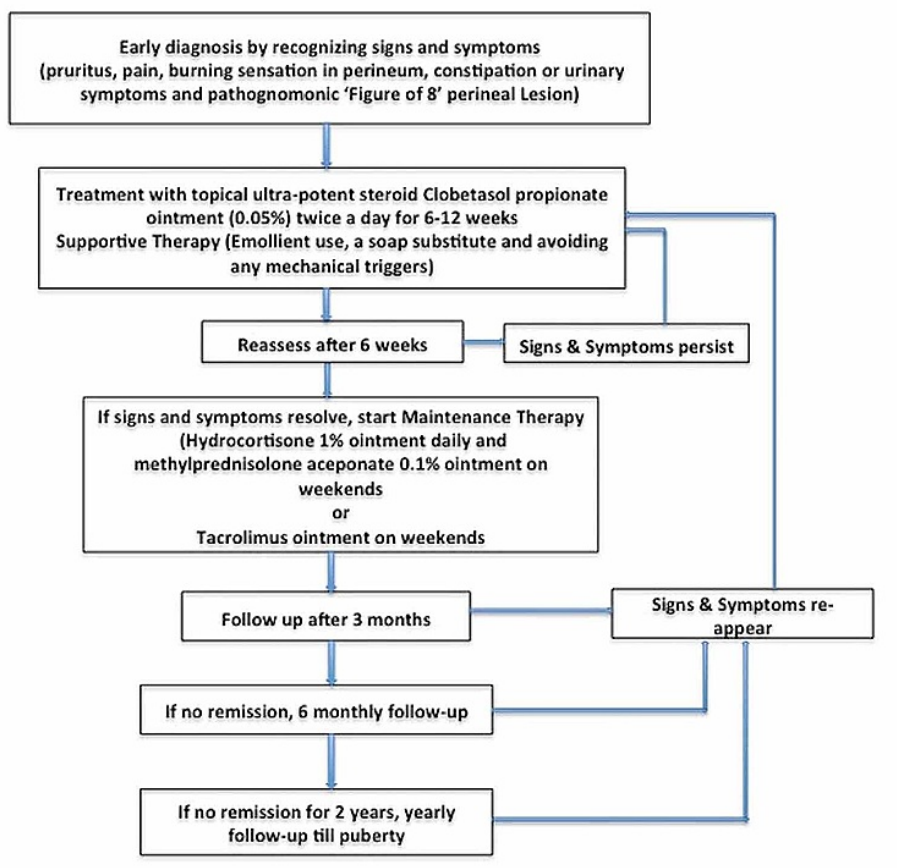

FIGURE 2: Suggested Management of Pediatric Vulvar Lichen Sclerosus

\section{Patient counseling and vulvar hygiene}

Patient should be counseled to avoid using soap and use emollients instead. Drying of skin after passing urine to prevent its contact is useful. Using a moisturizer or paraffin as a barrier cream is protective against exposure to urine. Lubricants and, if required, vaginal dilators may aid if intercourse is painful, which may happen with VLS. Light, soft, airy, and frictionless materials for undergarments like Derma silk etc. have been shown to improve the symptoms and are recommended. Patients should be counseled regarding the risk of developing vulvar cancer and, therefore, the need of self-examination to notice any change in skin color, texture, or development of ulcers etc. Asymptomatic LS should also be treated in an attempt to decrease the risk of progression to vulvar SCC(7). Women should be encouraged to examine their vulva for any changes, though this may be difficult in older women with co-morbidities such as osteoarthritis, visual problems, and obesity. Patients should be advised against smoking as it increases the risk of vulvar cancer.

It is important to emphasize that LS needs to be treated even if asymptomatic to prevent scarring leading to sexual and urinary problems. While treating, we need to comment that follow-up should initially be every three months to assess response - decrease in itching and pain. One needs to ensure that there is no atrophy of the skin because of treatment with steroids.

\section{Follow-up, prognosis, and recurrence}

Regular follow-up is necessary as there is an increased risk of developing SCC. However, there is no consensus between frequency and duration of follow-up. Follow-up may be done every three to six months for the first two years and then at least yearly to ensure adequacy of treatment and encourage compliance [5].

The features that need to be looked upon on follow-up visits includes compliance of treatment, symptomatic response, objective clinical response to treatment, need of maintenance therapy, development of adhesions or scarring, adverse effects of treatment, and development of SCC or vulvar intraepithelial neoplasia [5]. Long-term follow-up in specialist clinics is recommended for women who have persistent complaints, thickened skin, or history of neoplastic lesion.

\section{Conclusions}

VLS is a chronic inflammatory disorder, which affects women of all ages. Highly potent topical corticosteroids are the first-line treatment. However, their long-term use includes skin thinning. Side effects with local steroids are rare, and it is usually a well-tolerated therapy. Most of the second-line therapies are in experimental phases, and further data is required. Follow-up in these women is essential as there is a likely risk of malignant transformation in around $4 \%$ women. 


\section{Additional Information \\ Disclosures}

Conflicts of interest: In compliance with the ICMJE uniform disclosure form, all authors declare the following: Payment/services info: All authors have declared that no financial support was received from any organization for the submitted work. Financial relationships: All authors have declared that they have no financial relationships at present or within the previous three years with any organizations that might have an interest in the submitted work. Other relationships: All authors have declared that there are no other relationships or activities that could appear to have influenced the submitted work.

\section{References}

1. Bleeker MCG, Visser PJ, Overbeek LIH, van Beurden M, Berkhof J: Lichen sclerosus: incidence and risk of vulvar squamous cell carcinoma. Cancer Epidemiol Biomarkers Prev. 2016, 25:1224-30. 10.1158/10559965.EPI-16-0019

2. Lynch PJ, Moyal-Barracco M, Scurry J, Stockdale C: 2011 ISSVD terminology and classification of vulvar dermatological disorders: an approach to clinical diagnosis. J Low Genit Tract Dis. 2012, 16:339-44. 10.1097/LGT.0b013e3182494e8c

3. Singh N, Ghatage P: Etiology, clinical features, and diagnosis of vulvar lichen sclerosus: a scoping review . Obstet Gynecol Int. 2020, 2020:7480754. 10.1155/2020/7480754

4. Chi C, Kirtschig G, Baldo M, Brackenbury F, Lewis F, Wojnarowska F: Topical interventions for genital lichen sclerosus. Cochrane Database Syst Rev. 2011, 2011:CD008240. 10.1002/14651858.CD008240.pub2

5. Dalziel KL, Millard PR, Wojnarowska F: The treatment of vulval lichen sclerosus with a very potent topical steroid (clobetasol propionate 0.05\%) cream. Br J Dermatol. 1991, 124:461-4. 10.1111/j.13652133.1991.tb00626.x

6. Lee A, Bradford J, Fischer G: Long-term management of adult vulvar lichen sclerosus: a prospective cohort study of 507 women. JAMA Dermatol. 2015, 151:1061-7. 10.1001/jamadermatol.2015.0643

7. Kirtschig G, Becker K, Günthert A, et al.: Evidence-based (S3) guideline on (anogenital) lichen sclerosus . J Eur Acad Dermatol Venereol. 2015, 29:1-43. 10.1111/jdv.13136

8. Cooper SM, Gao XH, Powell JJ, Wojnarowska F: Does treatment of vulvar lichen sclerosus influence its prognosis?. Arch Dermatol. 2004, 140:702-6. 10.1001/archderm.140.6.702

9. Bradford J, Fischer G: Long-term management of vulval lichen sclerosus in adult women . Aust N Z J Obstet Gynaecol. 2010, 50:148-52. 10.1111/j.1479-828X.2010.01142.x

10. Pérez-López FR, Ceausu I, Depypere H, et al.: EMAS clinical guide: vulvar lichen sclerosus in peri and postmenopausal women. Maturitas. 2013, 74:279-82. 10.1016/j.maturitas.2012.12.006

11. Vilmer C, Balloy B, Porcher R, et al.: Vulvar lichen sclerosus: effect of long-term topical application of a potent steroid on the course of the disease. Arch Dermatol. 2004, 140:709-12. 10.1001/archderm.140.6.709

12. Corazza M, Virgili A, Toni G, Borghi A: Mometasone furoate in the treatment of vulvar lichen sclerosus: could its formulation influence efficacy, tolerability and adherence to treatment?. J Dermatolog Treat. 2018, 29:305-9. 10.1080/09546634.2017.1360990

13. Stücker M, Grape J, Bechara FG, Hoffmann K, Altmeyer P: The outcome after cryosurgery and intralesional steroid injection in vulvar lichen sclerosus corresponds to preoperative histopathological findings. Dermatology. 2005, 210:218-22. 10.1159/000083513

14. Ventolini G, Swenson KM, Galloway ML: Lichen sclerosus: a 5-year follow-up after topical, subdermal, or combined therapy. J Low Genit Tract Dis. 2012, 16:271-4. 10.1097/LGT.0b013e31823da7e8

15. Assmann T, Becker-Wegerich P, Grewe M, Megahed M, Ruzicka T: Tacrolimus ointment for the treatment of vulvar lichen sclerosus. J Am Acad Dermatol. 2003, 48:935-7. 10.1067/mjd.2003.8

16. Böhm M, Frieling U, Luger TA, Bonsmann G: Successful treatment of anogenital lichen sclerosus with topical tacrolimus. Arch Dermatol. 2003, 139:922-4. 10.1001/archderm.139.7.922

17. Funaro D, Lovett A, Leroux N, Powell J: A double-blind, randomized prospective study evaluating topical clobetasol propionate $0.05 \%$ versus topical tacrolimus $0.1 \%$ in patients with vulvar lichen sclerosus. J Am Acad Dermatol. 2014, 71:84-91. 10.1016/j.jaad.2014.02.019

18. Goldstein A, Creasey A, Pfau R, Phillips D, Burrows LJ: A double-blind, randomized controlled trial of clobetasol versus pimecrolimus in patients with vulvar lichen sclerosus. J Am Acad Dermatol. 2011, 64:99104. 10.1016/j.jaad.2010.06.011

19. Hengge U, Krause W, Hofmann H, et al.: Multicentre, phase II trial on the safety and efficacy of topical tacrolimus ointment for the treatment of lichen sclerosus. Br J Dermatol. 2006, 155:1021-8. 10.1111/j.13652133.2006.07446.x

20. Anderson K, Ascanio NM, Kinney MA, Krowchuk DP, Jorizzo JL: A retrospective analysis of pediatric patients with lichen sclerosus treated with a standard protocol of class I topical corticosteroid and topical calcineurin inhibitor. J Dermatolog Treat. 2016, 27:64-6. 10.3109/09546634.2015.1054777

21. Bracco GL, Carli P, Sonni L, et al.: Clinical and histologic effects of topical treatments of vulval lichen sclerosus. A critical evaluation. J Reprod Med. 1993, 38:37-40.

22. Sideri M, Origoni M, Spinaci L, Ferrari A: Topical testosterone in the treatment of vulvar lichen sclerosus . Int J Gynaecol Obstet. 1994, 46:53-6. 10.1016/0020-7292(94)90309-3

23. Virgili A, Corazza M, Bianchi A, et al.: Open study of topical $0.025 \%$ tretinoin in the treatment of vulvar lichen sclerosus. One year of therapy. J Reprod Med. 1995, 40:614-8.

24. Borghi A, Corazza M, Minghetti S, Virgili A: Topical tretinoin in the treatment of vulvar lichen sclerosus: an advisable option?. Eur J Dermatol. 2015, 25:404-9. 10.1684/ejd.2015.2595

25. Origoni M, Ferrari D, Rossi M, Gandini F, Sideri M, Ferrari A: Topical oxatomide: an alternative approach for the treatment of vulvar lichen sclerosus. Int J Gynaecol Obstet. 1996, 55:259-64. 10.1016/s00207292(96)02768-3

26. Shelley WB, Shelley ED, Amurao CV: Treatment of lichen sclerosus with antibiotics . Int J Dermatol. 2006, 
45:1104-6. 10.1111/j.1365-4632.2006.02978.x

27. Goldstein AT, Burrows LJ, Belkin ZR, Pfau R, Bremmer M, Goldfinger C, Dreher F: Safety and efficacy of human fibroblast lysate cream for vulvar lichen sclerosus: a randomized placebo-controlled trial. Acta Derm Venereol. 2015, 95:847-9.

28. Virgili A, Minghetti S, Borghi A, Corazza M: Long-term maintenance therapy for vulvar lichen sclerosus: the results of a randomized study comparing topical vitamin E with an emollient. Eur J Dermatol. 2013, 23:18994. 10.1684/ejd.2013.1987

29. Balchander D, Nyirjesy P: Fractionated CO2 laser as therapy in recalcitrant lichen sclerosus . J Low Genit Tract Dis. 2020, 24:225-8. 10.1097/LGT.0000000000000512

30. Hobson JG, Ibrahim SF, Mercurio MG: Recalcitrant vulvar lichen sclerosus treated with erbium YAG laser . JAMA Dermatol. 2019, 155:254-6.

31. Terras S, Gambichler T, Moritz RKC, Stücker M, Kreuter A: UV-A1 phototherapy vs clobetasol propionate, 0.05\%, in the treatment of vulvar lichen sclerosus: a randomized clinical trial. JAMA Dermatol. 2014, 150:621-7. 10.1001/jamadermatol.2013.7733

32. Li Z, Wang Y, Wang J, et al.: Evaluation of the efficacy of 5-aminolevulinic acid photodynamic therapy for the treatment of vulvar lichen sclerosus. Photodiagnosis Photodyn Ther. 2020, 29:101596. 10.1016/j.pdpdt.2019.101596

33. Lan T, Zou Y, Hamblin MR, Yin R: 5-Aminolevulinic acid photodynamic therapy in refractory vulvar lichen sclerosus et atrophicus: series of ten cases. Photodiagnosis Photodyn Ther. 2018, 21:234-8. 10.1016/j.pdpdt.2017.12.003

34. Shi L, Miao F, Zhang LL, et al.: Comparison of 5-aminolevulinic acid photodynamic therapy and clobetasol propionate in treatment of vulvar lichen sclerosus. Acta Derm Venereol. 2016, 15:684-8. 10.2340/000155552341

35. Hillemanns P, Untch M, Pröve F, Baumgartner R, Hillemanns M, Korell M: Photodynamic therapy of vulvar lichen sclerosus with 5-aminolevulinic acid. Obstet Gynecol. 1999, 93:71-4. 10.1016/s0029-7844(98)00321-4

36. Kastner U, Altmeyer P: [Cryosurgery--the last resort or a surgical alternative in the treatment of lichen sclerosus et atrophicus of the vulva (LSAV)?]. J Dtsch Dermatol Ges. 2003, 1:206-11. 10.1046/j.16100387.2003.03517.x

37. Boero V, Brambilla M, Sipio E, et al.: Vulvar lichen sclerosus: A new regenerative approach through fat grafting. Gynecol Oncol. 2015, 139:471-5. 10.1016/j.ygyno.2015.10.014

38. Almadori A, Hansen E, Boyle D, et al.: Fat grafting improves fibrosis and scarring in vulvar lichen sclerosus: results from a prospective cohort study. J Low Genit Tract Dis. 2020, 24:305-310. 10.1097/LGT.0000000000000520

39. Eshtiaghi P, Sadownik LA: Fact or fiction? Adipose-derived stem cells and platelet-rich plasma for the treatment of vulvar lichen sclerosus. J Low Genit Tract Dis. 2019, 23:65-70. 10.1097/LGT.00000000000000440

40. Borghi A, Corazza M, Minghetti S, Toni G, Virgili A: Avocado and soybean extracts as active principles in the treatment of mild-to-moderate vulvar lichen sclerosus: results of efficacy and tolerability. J Eur Acad Dermatol Venereol. 2015, 29:1225-30. 10.1111/jdv.12617

41. Kelly RA, Foster DC, Woodruff JD: Subcutaneous injection of triamcinolone acetonide in the treatment of chronic vulvar pruritus. Am J Obstet Gynecol. 1993, 169:568-70. 10.1016/0002-9378(93)90622-p

42. Bousema MT, Romppanen U, Geiger JM, Baudin M, Vähä-Eskeli K, Vartiainen J, Vuopala S: Acitretin in the treatment of severe lichen sclerosus et atrophicus of the vulva: a double-blind, placebo-controlled study. J Am Acad Dermatol. 1994, 30:225-31. 10.1016/s0190-9622(94)70021-4

43. Tang GX, Wu X, Chen JP, Zhou BS: [Study on the risk factors of 100 cases with vulvar dystrophy] . Zhonghua Liu Xing Bing Xue Za Zhi. 2003, 24:932-4.

44. Vieira BP, Lima SJ, Cavaco-Gomes J, Beires J, Martinez-de-Oliveira J: What differentiates symptomatic from asymptomatic women with lichen sclerosus?. Gynecol Obstet Invest. 2015, 79:263-8. 10.1159/000367788

45. Chmel R, Novackova M, Fait T, Zámečník L, Krejčová L, Pastor Z: Clitoral Phimosis: Effects on Female Sexual Function and Surgical Treatment Outcomes. J Sex Med. 2019, 16:257-66. 10.1016/j.jsxm.2018.12.012

46. Wyman AM, Lafranchise E, Lynch C: External use of an oxidized regenerated cellulose agent in the treatment of labial agglutination in postmenopausal women. Obstet Gynecol. 2018, 132:1238-40. 10.1097/AOG.0000000000002919

47. Abramov Y, Elchalal U, Abramov D, Goldfarb A, Schenker JG: Surgical treatment of vulvar lichen sclerosus: a review. Obstet Gynecol Surv. 1996, 51:193-9. 10.1097/00006254-199603000-00023

48. Virgili A, Minghetti S, Borghi A, Corazza M: Proactive maintenance therapy with a topical corticosteroid for vulvar lichen sclerosus: preliminary results of a randomized study. Br J Dermatol. 2013, 168:1316-24. 10.1111/bjd.12273

49. Garzon MC, Paller AS: Ultrapotent topical corticosteroid treatment of childhood genital lichen sclerosus . Arch Dermatol. 1999, 135:525-8. 10.1001/archderm.135.5.525

50. Li Y, Xiao Y, Wang H, Li H, Luo X: Low-concentration topical tacrolimus for the treatment of anogenital lichen sclerosus in childhood: maintenance treatment to reduce recurrence. J Pediatr Adolesc Gynecol. 2013, 26:239-42. 10.1016/j.jpag.2012.11.010 